\title{
Imprinting bias stress in functional composites
}

\author{
V. Khovaylo \\ Institute of Radioengineering and Electronics of RAS, Moscow 125009, Russia and \\ Physics Department, Moscow State Mining University, Moscow 119991, Russia \\ V. Koledov, E. Perov, and V. Shavrov \\ Institute of Radioengineering and Electronics of RAS, Moscow 125009, Russia \\ G. Lebedev and D. Zakharov \\ State Technological University "Moscow Institute for Steel and Alloys", Moscow 119991, Russia \\ M. Ohtsuka \\ Institute of Multidisciplinary Research for Advanced Materials, Tohoku University, Sendai 980-8577, Japan \\ V. Pushin \\ Institute of Metal Physics, Ekaterinburg 620041, Russia \\ H. Miki and T. Takagi \\ Institute of Fluid Science, Tohoku University, Sendai 980-8577, Japan
}

(Dated: October 29, 2018)

\begin{abstract}
We propose a simple yet effective method which allows one to attain large reversible shape changes in shape memory bimetallic composites without training procedure. It is based on the conservation of strongly anisotropic martensite microstructure artificially created in the shape memory layer. This procedure results in appearance of stress field when the shape memory layer is transformed to the austenitic state which brings about two-way shape memory effect. Utilization of this method for preparation of TiNi-based composite with a thickness of $60 \mu \mathrm{m}$ allowed us to achieve $0.9 \%$ reversible bending deformation. It is also suggested that the implementation of this method during preparation of piezoelectric or magnetostrictive composites permits to imprint bias stress and thus to improve their characteristics without use of an external load.
\end{abstract}

PACS numbers:

The ability of shape memory materials to the reversible spontaneous shape change - two-way shape memory effect - has widely been used for various applications such as microactuators or micromachines in microelectromechanical systems (MEMS), various actuators in electrical appliances, medical implants and guidewires in biomedical engineering etc. However, this unique property is not intrinsic to a shape memory material. It is generally acknowledged that the two-way shape memory effect is due to the relaxation of residual stresses which promotes formation of specific martensite variants upon transformation to the low-temperature state. To create internal stress fields in the austenitic state, somewhat complicated training procedures such as introduction of plastic deformation, constraint aging, thermal cycling, or utilization of precipitates has to be performed [1, 2]. This is inconvenient from technological point of view and increases the total cost of devises. Moreover, training also results in concomitant effects, e.g. changes of transformation temperature and hysteresis.

Here we propose a simple yet effective method which allows one to bring about the two-way shape memory effect in shape memory bimetallic composites without training procedure. The essence of the proposed method is that a shape memory layer is bonded with a metallic layer, as in conventional bimetallic plate, but prior to the bonding the shape memory layer is stressed in the martensitic state. Preliminary stressing, particularly tension, creates strongly anisotropic martensite microstructure which then conserved by a bonding with the metallic layer.

The appearance of the driving force underlying twoway shape memory effect in thus prepared composites is schematically illustrated in Fig. 1. When the shape memory layer is cooled down below martensite start temperature $M_{s}$, a specific microstructure consisting of differently oriented martensitic variants is formed (Fig. 1a). An external load easily changes this microstructure, promoting growth of the favorably oriented martensitic variants and thus leading to seemingly plastic deformation $\delta l$ (Fig. 1b). If the shape memory layer is bonded to a metallic layer (Fig. 1c) its strongly anisotropic microstructure will be conserved. Upon heating above the reverse martensitic transformation temperature $A_{f}$ the martensitic variants disappear and the shape memory layer restores its original dimensions. Due to the bonding with the metallic layer, this leads to a strong bending deformation of this bimetallic composite (Fig. 1d). In the 


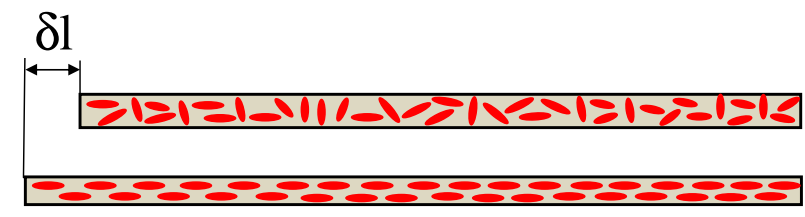

a)
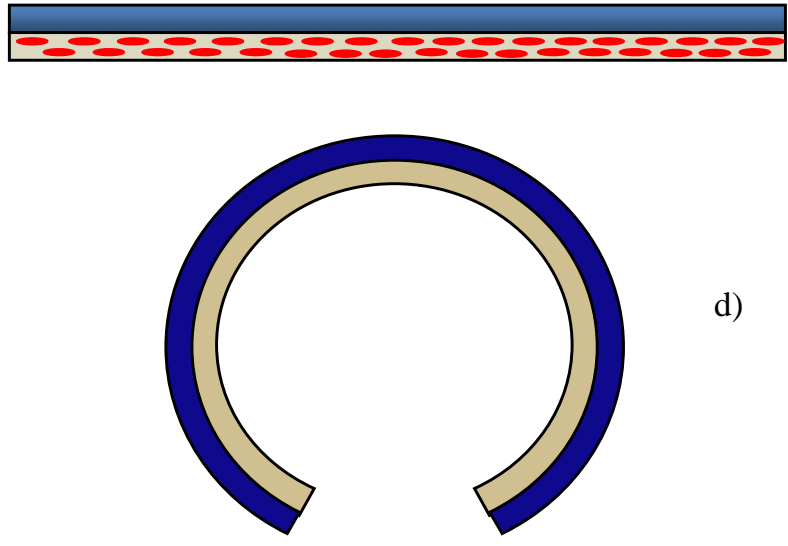

-

e)

FIG. 1: Training-free two-way shape memory effect in a bimetallic composite. Martensitic variants (schematically shown by ellipses) formed in a shape memory layer after transformation from the austenitic state (a); Anisotropic martensitic microstructure created by an external stress (b); Orientation of the martensitic variants in the shape memory layer imprinted by bonding with a metallic layer (c); Two-way shape memory effect in the bimetallic composite: deformation in the austenitic state $(\mathrm{d})$ and the reversion of the original shape upon transition to the martensitic state (e).

martensite nucleation process occurring upon cooling below $M_{s}$ temperature, relaxation of the bending-induced residual stress field selects certain martensitic variant and the bimetallic composite reverts its low-temperature flat shape (Fig. 1e). Since the metallic layer causes recurrent stress field in the austenitic state of the shape memory layer the bimetallic composite exhibits two-way shape memory effect.

Experimental verification of this method was realized using $\mathrm{Ti}_{50} \mathrm{Ni}_{25} \mathrm{Cu}_{25}$ rapidly quenched ribbons $\left(M_{s}=\right.$ $336 \mathrm{~K}$ ) with thickness of $30 \mu \mathrm{m}$ which served as a shape memory layer. The pseudoplastic deformation $\delta l \approx 1 \%$ was produced in the ribbon by cooling down though the martensitic transformation temperature under an external load. After that the $\mathrm{Ti}_{50} \mathrm{Ni}_{25} \mathrm{Cu}_{25}$ ribbon was glued to a stainless ribbon of the same thickness. Subsequent experiment showed that the bimetallic composite exhibits reversible bending/unbending upon heating/cooling. Similar results were obtained for prepared in the same manner bimetallic composite made of a $\mathrm{Ni}_{49.5} \mathrm{Mn}_{28} \mathrm{Ga}_{22.5}$ thin film $\left(M_{s}=309 \mathrm{~K}\right)$ and a stainless ribbon, both of $5 \mu \mathrm{m}$ thickness.

Gluing of the constituting layers of the bimetallic composites has significant shortcoming since their reversible deformation degrades rather rapidly due to the poor adhesion between the individual layers. In another composite, a $30 \mu \mathrm{m}$ Ni layer was electrolytically deposited at room temperature onto the surface of $\mathrm{Ti}_{50} \mathrm{Ni}_{25} \mathrm{Cu}_{25}$ ribbon thermomechanically treated in the same way as described above. In order to evaluate performance of the $\mathrm{Ti}(\mathrm{NiCu}) / \mathrm{Ni}$ composite prepared by the electrolytical deposition, it was subjected to thermocycling through the martensitic transition temperature. Curvature radius $R$ of the composite in the austenitic state and the corresponding bending deformation $\varepsilon=h / R$ (where $h$ is the thickness of the composite) has been measured as a function of number of thermocycles $N$. The results of this experiment (Fig. 2) have revealed that the bimetallic composite shows $\varepsilon \approx 0.9 \%$ upon the first heating/cooling cycle and exhibits fairly stable performance at least up to 2000 thermocycles through the martensitic transition temperature. Observed decrease of with the increase in the number of cycles $N$ is presumably caused by the accumulation of inelastic defects in the Ni layer.

It has also to be noted that, at the beginning of the

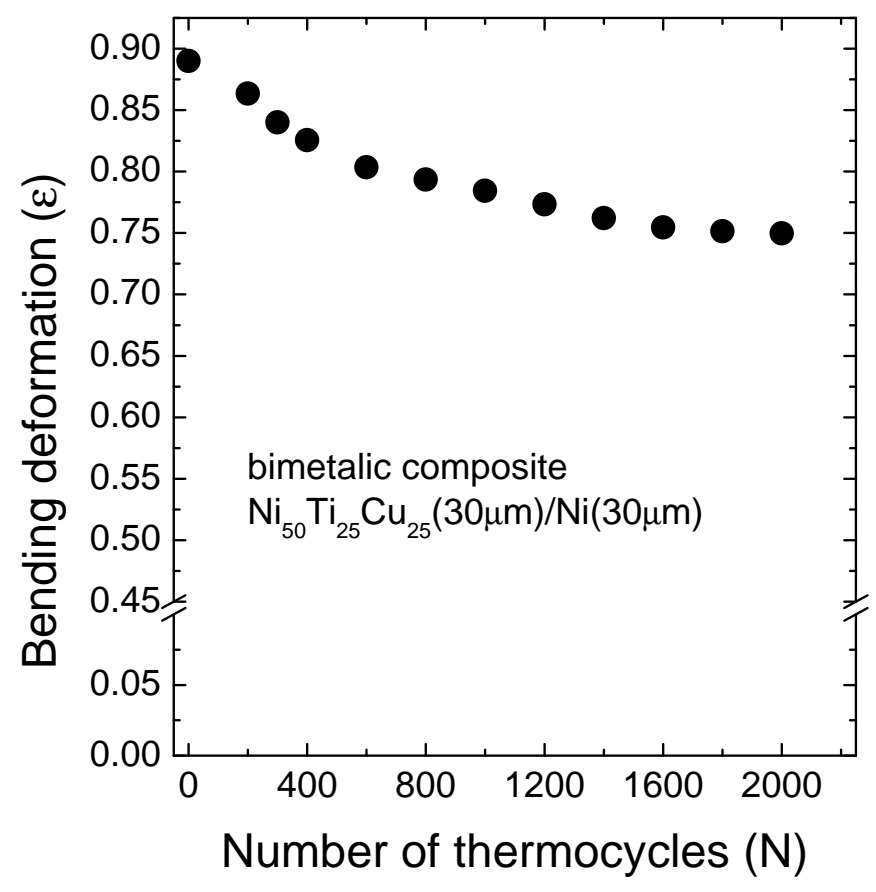

FIG. 2: Reversible bending deformation as a function of number of thermocycles $N$ through the martensitic transition temperature in a shape memory-based bimetallic composite prepared by room-temperature electrolytical deposition of a $30 \mu \mathrm{m}$ Ni layer onto a

$\mathrm{Ti}_{50} \mathrm{Ni}_{25} \mathrm{Cu}_{25}$ ribbon of the same thickness. 
experiment (up to first 400 thermocycles), the composite did not completely recover its shape in the martensitic state. This presumably means that the anisotropic distribution of the martensitic variants created by the external load (Fig. 1b) was not perfectly conserved by the Ni layer due to rather a large thickness of the $\mathrm{Ti}_{50} \mathrm{Ni}_{25} \mathrm{Cu}_{25}$ ribbon. As a result, at the initial stage of the experiment the nucleation of randomly oriented martensitic variants took place in lesser strained areas of the composite. However, it is evident that characteristics of the composites can be improved by a proper choice of processing method, adjustment of the thickness of the constituting layers, and selection of the metallic layer with suitable Young's modulus and elastic properties.

It must be emphasized that our approach essentially differs from those reported so far in the literature (see for review Refs. [3-6]). Studies of shape memory thin films deposited onto various substrates have revealed that they frequently exhibit a two-way shape memory behavior [713]. Observed in these composites reversible bending deformations originate from stress fields which are induced by a lattice mismatch between the film and substrate. In our approach, a stress field in the austenitic state of a shape memory layer is created by the anisotropic martensite microstructure; the role of the lattice mismatch is immaterial. This mechanism allows preparation of composites with bending deformations comparable to the superelastic strains which can be as large as $6 \%$ in TiNi thin films [14]. Other advantage of this method is that there is no strict limitation on the thickness of the constituting layers.

We suggest that the proposed method of imprinting bias stress can also be used to enhance characteristics of piezoelectric and magnetostrictive composites. Indeed, it is well documented that striction of electro- or magnetostrictive materials is markedly enhanced when they are subjected to mechanical loading which provides a direction for preferential orientation of structural domains [15] or magnetization vector [16]. For thin enough samples, anisotropic microstructure created by electric or magnetic field can be easy conserved by a deposition of a metallic layer. For this aim the deposition should be done in the presence of the field. This method has an additional advantage for miniaturization of such devises since no mechanical parts are used for the application of bias stress.

In conclusion, we have proposed and experimentally verified that in shape memory-based composites a strongly anisotropic martensite microstructure can be preserved by a deposition of a metallic layer. This results in large bending deformations of composites comparable to the superelastic strain of the shape memory layer. We also have proposed that this approach is capable to improve characteristics of piezoelectric and magnetostrictive composites.

[1] K. Otsuka and C. M. Wayman (eds) Shape Memory Materials (Cambridge University Press, 1998).

[2] X. M. Zhang, J. Fernandez, and J. M. Guilemany, Mater. Sci. Eng. A 438-440, 431 (2006).

[3] Z. G. Wei, R. Sandstrom, and S. Miyazaki, J. Mater. Sci. 33, 3763 (1998).

[4] Y. Fu, H. Du, W. Huang, S. Zhang, and M. Hu, Sensors Actuators A 112, 395 (2004).

[5] B. Winzek, S. Schmitz, H. Rumpf, T. Sterzl, R. Hassdorf, S. Thienhaus, J. Feydt, M. Moske, and E. Quandt, Mater. Sci. Eng. A 378, 40 (2004).

[6] S. A. Wilson, et al., Mater. Sci. Eng. R 56, 1 (2007).

[7] E. Quandt, C. Halene, H. Holleck, K. Feit, M. Kohl, P. Schloßmacher, A. Skokan, and K. D. Skrobanck, Sensors Actuators A 53, 434 (1996).

[8] B. Winzek and E. Quandt, Z. Metallkd. 90, 796 (1999).

[9] A. L. Roytburd, T. S. Kim, Q. Su, J. Slutsker, and M. Wuttig, Acta Mater. 46, 5095 (1998).

[10] H. Rumpf, C. M. Craciunescu, H. Modrow, Kh. Olimov, E. Quandt, and M. Wuttig, J. Magn. Magn. Mater. 302, 421 (2006).

[11] W. M. Huang, Q. Y. Liu, L. M. He, and J. H. Yeo, Sensors Actuators A 114, 118 (2004).

[12] A. Camposeo, N. Puccini, F. Fuso, M. Allegrini, E. Arimondo, and A. Tuissi, Appl. Sur. Sci. 208-209, 518 (2003).

[13] T. M. Adams, S. R. Kirkpatrick, Z. Wang, and A. Siahmakoun, Mater. Lett. 59, 1161 (2005).

[14] A. Ishida, M. Sato, A. Takei, and S. Miyazaki, Mater. Trans., JIM 36, 1349 (1995).

[15] E. Burcsu, G. Ravichandran, and K. Bhattacharya, Appl. Phys. Lett. 77, 1698 (2000).

[16] A. E. Clark, J. P. Teter, and O. D. McMasters, J. Appl. Phys. 63, 3910 (1988). 